\title{
Ultrasonido endobronquial más aspiración con aguja fina y evaluación patológica rápida en sala. Medicina de precisión y trabajo multidisciplinario: serie de casos
}

\author{
Endobronchial ultrasound-guided transbronchial \\ needle aspiration and rapid on-site pathologic \\ evaluation. Medicine of presicion and multidisciplinary \\ work: case series
}

Liliana Fernández, MD. ${ }^{(1)}$; Luz Fernanda SuA, MD., PhD. ${ }^{(2)}$; MAuricio Velásquez, MD. ${ }^{(3)}$

\begin{abstract}
Resumen
El ultrasonido endobronquial más aspiración con aguja fina (EBUS-TBNA, su sigla en inglés por endobronchial ultrasound-guided transbronchial needle aspiration) desempeña un rol importantísimo en el diagnóstico de las lesiones mediastinales, así como tambien en la estadificación y reestadificación del cáncer pulmonar. Requiere evaluación multidisciplinaria inicial y análisis de las imágenes, del estado general del paciente, así como de los riesgos y beneficios, además de un trabajo estrecho con el grupo de patología, que efectúa una evaluación patológica rápida en sala (ROSE, su sigla en inglés por rapid on-site pathologic evaluation) con el obejtivo de mejorar el rendimiento diagnóstico. Este último fue del 78\% con coloración de Diff-Quick y se incrementó con bloque celular y técnicas adicionales de patología molecular a 89\%, la mediastinoscopia diagnóstica en 7\%, la rentabilidad diagnóstica en $91 \%$ y la exactitud en carcinoma pulmonar de células no pequeñas (CPCNP), en un $97 \%$.
\end{abstract}

La retroalimentación inmediata en la sala por parte del patólogo, permite mejorar el diagnóstico y evaluar la calidad y cantidad de la muestra, trabajando así de forma multidisciplinaria. Se describe una serie de 54 casos de EBUS-TBNA con ROSE, pertenecientes a la Fundación Valle del Lili, Cali-Colombia.

Palabras clave: ultrasonido endobronquial, broncoscopia intervencionista, evaluación patológica rápida en sala.

\begin{abstract}
Endobronchial ultrasound-guided transbronchial needle aspiration (EBUS-TBNA) plays a crucial role in diagnosing mediastinal lesions, as well as in staging and restaging lung cancer. It requires a multidisciplinary initial approach and analysis of image studies, of the patient's general condition, and of risks and benefits, besides close collaboration with the pathology team, who carry out rapid on-site pathologic evaluation (ROSE), with the purpose of improving the diagnostic yield. The latter was $78 \%$ with Diff-Quick staining, and increased to $89 \%$ with cell block and additional molecular pathology techniques, $7 \%$ with diagnostic mediastinoscopy, $91 \%$ diagnostic profitability, and 97\% accuracy in non-small-cell lung cancer.
\end{abstract}

Immediate on-site feedback by the pathologist allows for improving diagnosis and assessing the quality and quantity of the sample (multidisciplinary collaboration). We describe a series of 54 cases of EBUS-TBNA with ROSE, from the Fundación Valle del Lili, Cali-Colombia.

Keywords: endobronchial ultrasound, interventional bronchoscopy, rapid on-site pathologic evaluation.

\begin{abstract}
${ }^{(1)}$ Especialista en Medicina Interna. Subespecialista en Neumología. Neumología Intervencionista. Grupo de Investigación Biomédica en Tórax. Fundación Valle del Lili. Universidad ICESI, Facultad de Ciencias de la Salud. Cali, Colombia. ${ }^{(2)}$ Especialista en Anatomía Patológica y Patología Clínica. Patología Pulmonar. Ciencias Biomédicas. Departamento de Patología y Medicina de Laboratorio. Laboratorio de Patología Molecular. Grupo de Investigación Biomédica en Tórax. Fundación Valle del Lili. Universidad ICESI, Facultad de Ciencias de la Salud. Cali, Colombia.

${ }^{(3)}$ Especialista en Cirugía General. Subespecialista en Cirugía de Tórax. Grupo de Investigación Biomédica en Tórax. Fundación Valle del Lili. Universidad ICESI, Facultad de Ciencias de la Salud. Cali, Colombia.

Correspondencia: Liliana Fernández, correo electrónico: 1fernandez@fcvl.org Recibido: 14/11/14, Aceptado: 10/12/14.
\end{abstract}




\section{Introducción}

El EBUS-TBNA es una técnica broncoscópica que permite la visualización por ultrasonido de la pared traqueobronquial y las estructuras adyacentes, así como del parénquima pulmonar. Sirve para valorar el mediastino, las lesiones peribronquiales y paratraqueales así como los nódulos linfáticos en tiempo real y facilita la toma de muestras, las cuales pueden ser analizadas en sala por el patólogo intervencionista a fin de mejorar aún más el rendimiento diagnóstico (1-3).

El EBUS lineal (figura 1) es un equipo que tiene un diámetro mayor que los broncoscopios convencionales, alrededor de $7 \mathrm{~mm}$, por lo cual generalmente se introduce a través de la boca, bajo sedación profunda o anestesia general usualmente mediante máscara laríngea o tubo orotraqueal o transoral. Tiene un canal de examen de $2 \mathrm{~mm}$ por el cual se avanzan agujas 19-22 G. Su campo de visión es oblicuo, entre $35-45^{\circ}$, de modo que usualmente antes del procedimiento se ha debido realizar una broncoscopia diagnóstica para evaluar detalladamente la vía aérea. Posee un transductor convexo incorporado en la punta, especialmente diseñado, que emite un haz de ultrasonidos paralelo al eje mayor del endoscopio de 50 a $90^{\circ}$, con frecuencias entre 5 y $12 \mathrm{MHz}$. Con las frecuencias altas se evalúan muy bien las capas

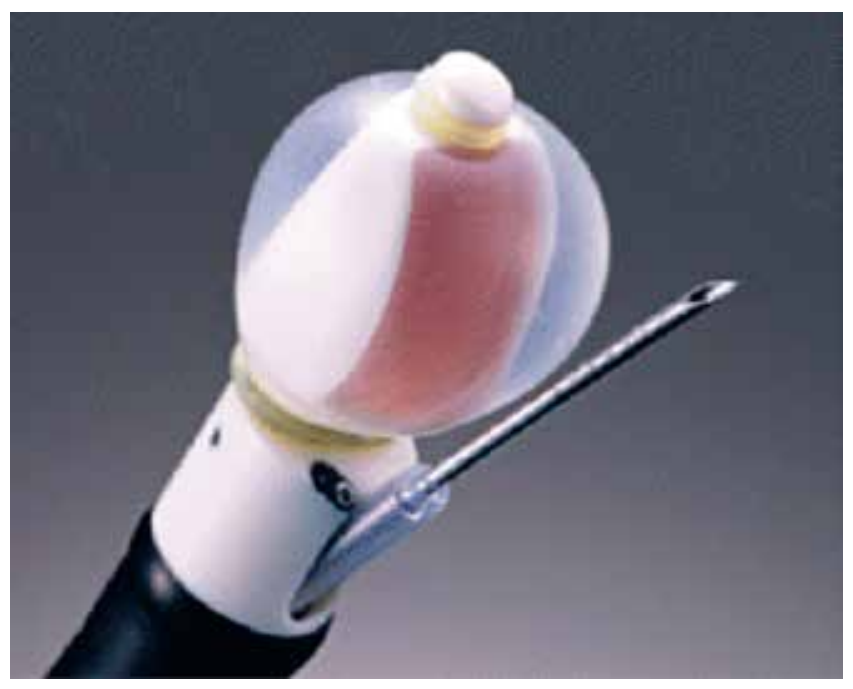

Figura 1. Ultrasonido endobronquial (cortesía de Olympus). menos profundas, lo cual es ideal para detectar lesiones mediastinales. Está provisto de Doppler color pulsado para detectar flujo sanguíneo y tiene un procesador que da la imagen de videobroncoscopia y al mismo tiempo la imagen de ultrasonido y Doppler pulsado, lo cual garantiza la exactitud en la punción. Para mejorar la imagen, está dotado de un balón distal que se infla cuando se identifican las estaciones ganglionares o las lesiones a estudiar $(4,5)$.

$\mathrm{Su}$ ventaja radica en poder efectuar punción dirigida en tiempo real. La detección de adenopatías por EBUS es posible incluso con tamaños ganglionares de 5-10 mm. Las estaciones ganglionares a las que es posible acceder son: 1, 2, 3, 4, 7, 10, 11 y 12 (6). Sin embargo, es necesario hacer una planeación previa del examen teniendo en cuenta las imágenes de la escanografía (TAC) y/o la tomografía por emisión de positrones (PET) y diseñando una ruta, pues la punción de las estaciones debe ser secuencial, es decir, se empieza por las más altas para realizar una verdadera estadificación tumoral (4-6).

Se trata de una técnica mínimamente invasiva ambulatoria, que usualmente necesita sedación profunda que puede ser administrada por el operador pero en la que, en la mayoría de casos, se prefiere la intervención del grupo de anestesia. Es secuencial y ordenada y en cada estación se hacen cuantos pases se requieran para garantizar el estudio adecuado del ganglio, usualmente 4 a 5 por estación. En este punto se resalta la importancia del acompañamiento del patólogo en sala. Así mismo la prueba requiere entrenamiento específico y de no aplicarse con criterio clínico razonado, puede enlentecer, complicar o encarecer el proceso de diagnóstico y la estadificación (4-6).

Por mucho tiempo la anatomía patológica y la patología clínica fueron consideradas especialidades de escritorio y laboratorio. La patología moderna ha virado involucrándose en grupos de trabajo multidisciplinarios conformados para tomar decisiones terapéuticas y quirúrgicas en salas de cirugía, endoscopia o imágenes diagnósticas.

Por su parte, la patología intervencionista permite explorar técnicas para mejorar la cantidad y 
calidad de las muestras, precisar los diagnósticos, disminuir las intervenciones y efectuar estudios complementarios de patología molecular tan relevante hoy en el pronóstico y en la elección de esquemas de tratamiento (medicina personalizada), como es el caso del carcinoma de células no pequeñas (CPCNP) en el que se precisa el estudio de la mutación del gen EGFR exón 18, 19, 20 y 21 y el estudio de translocaciones del gen EML4-ALK. Este modelo de trabajo en patología fue propuesto por Louis et al. (7), e involucra especialización y equipos de trabajo multidisciplinarios.

La implementación de ROSE durante el EBUSTBNA, disminuye las tasas de reintervención al confirmar que se han obtenido muestras de buena calidad. El proceso diagnóstico puede tomar alrededor de 18 horas con técnicas de coloración básica como la hematoxilina y eosina (H\&E), en tanto que los estudios de patología molecular pueden requerir entre 24 y 36 horas en un centro de alto nivel como la Fundación Valle del Lili.

En este artículo se muestran algunos casos evaluados en dicha institución y se resalta la ventaja de la evaluación patológica en sala, de manera conjunta.

\section{Materiales y métodos}

Estudio prospectivo y descriptivo, llevado a cabo durante mayo de 2012 y diciembre de 2013, en el que se incluyeron pacientes que requerían EBUS para la evaluación de su condición, previa discusión en una junta de desiciones multidisciplinaria. Las indicaciones del EBUS para la serie de estudio fueron la estadificación de tumores primarios malignos pulmonares, el diagnóstico de masas pulmonares o mediastinales, las alteraciones de los ganglios linfáticos en tomografía axial computarizada (TAC) o en la tomografía por emeisión de positrones (PET/CT), la reestadificación en la evolución del cáncer pulmonar, la presencia de ganglios linfáticos iguales o mayores de $1 \mathrm{~cm}$ de diámetro y los casos de pacientes con limitación de su condición clínica que impedía la realización de procedimientos más invasivos para estudio. En total se intervinieron 54 pacientes cuyos procedimientos fueron hechos en el servicio de endosco- pia de la Fundación Valle del Lili, bajo sedación profunda y control anestésico. El EBUS se realizó con un equipo Olympus ${ }^{\circledR}$ bronchoscope + US probe +22 G FNA.

En este periodo se implementó el servicio de patología intervencionista y se dio a conocer en la institución la patología de evaluación en sala (ROSE). El equipo de trabajo constó de un carro de transporte (figura 2), un microscopio de luz y una batería de tinción con la coloración de Diff-Quick para los extendidos de diagnóstico en sala. Se realizaron pruebas especiales con fijadores celulares específi$\cos \left(\right.$ Transfix $\left.{ }^{\circledR}\right)$ para citometría de flujo de punciones ganglionares sospechosas de enfermedad linfoproliferativa o lavados broncoalveolares (BAL). El material celular extraído se fijó en formalina bufferada al $10 \%$ y los bloques celulares fueron fabricados en base de Agar al 6\%, lo que permitió mayor agregación y concentración celular.

En las muestras obtenidas para bloque celular $(n=54)$ se realizó la coloración básica de hematoxilina y eosina (H\&E), más coloraciones especiales para microorganismos como PAS, BK o Gomory (GMS), mientras que la técnica de inmunohistoquímica automatizada se hizo en el equipo Benchmark XT y GX de Ventana ${ }^{\circledR}$ en el que se usaron anticuerpos con expresión proteica para genes como el TTF-1, p63, Napsina A, Citoqueratina 5/6, Cromogranina, Sinaptofisina y CD56. La extracción de AND se realizó mediante técnica manual con el kit de Qiagen ${ }^{\circledR}$ o de

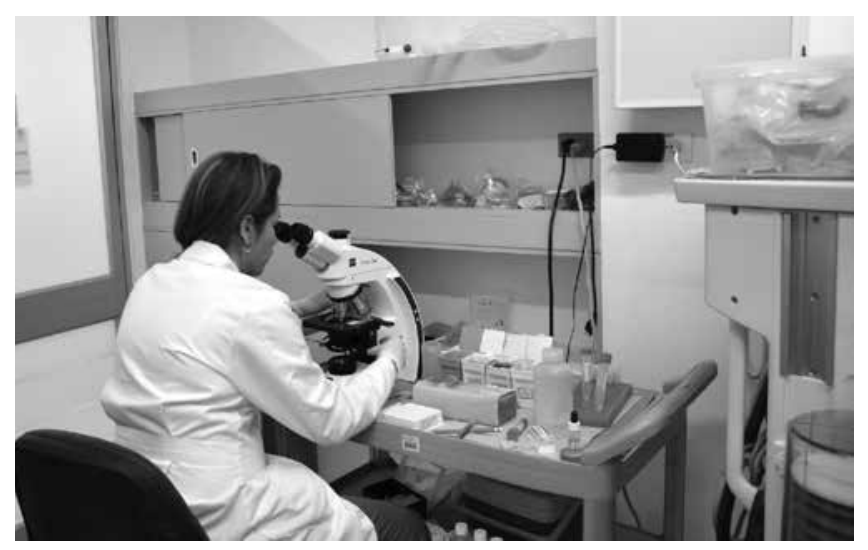

Figura 2. (ROSE) Evaluación en sala por citopatología. 
forma automatizada con el Sistema MagNa Lyser Instrument y MagNAPure ${ }^{\circledR}$ de Roche, con controles de calidad adecuados para el ADN extraído, de característica amplificable. Se realizaron estudios moleculares como la reacción en cadena de la polimerasa (PCR) para determinar la mutación del factor de crecimiento epidérmico (EGFR), exones 19 y 21 con el sistema 2100 Bioanalyzer $^{\circledR}$, cultivos microbiológicos y pruebas moleculares microbiológicas como el GeneXpert ${ }^{\circledR}$ System.

Todos los procedimientos diagnósticos se verificaron y aplicaron según los requerimientos para cada prueba en el laboratorio de patología molecular, citometría de flujo, laboratorio de genética molecular y laboratorio de microbiología molecular según los controles de calidad del Colegio Americano de Patólogos (CAP).

\section{Resultados y discusión}

El éxito en la implementación de este tipo de pruebas depende de factores como la disponibilidad del personal entrenado, los equipos y el tiempo necesario para la realización del procedimiento (figura 3), además de una unidad de broncoscopia y un departamento de patología y medicina de laboratorio que cuente con técnicas citológicas, histológicas y de patología molecular. Se analizaron los casos examinados y el diagnóstico en sala en cuanto a la

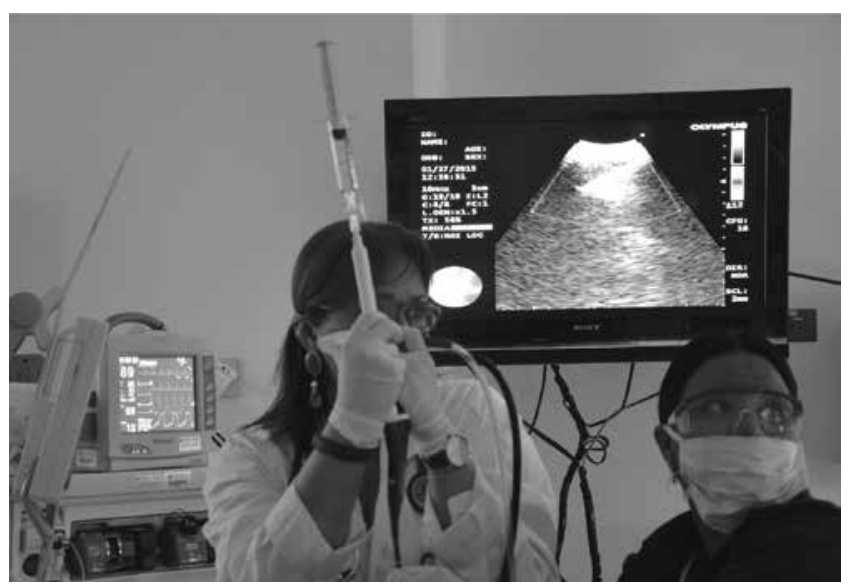

Figura 3. Ultrasonido endobronquial más aspiración con aguja fina (EBUS-TBNA); toma de muestra. adecuada representación de la lesión o localización anatómica, específicamente en la evaluación de las estaciones ganglionares. Al realizar esta comparación se encontró un ROSE del 78\% en los extendidos valorados solo con la coloración de Diff-Quick y se incrementó el porcentaje con el bloque celular y las técnicas adicionales a un $89 \%$ de rendimiento diagnóstico, contabilizando un mínimo de cinco pases por estación ganglionar o lesión tumoral, lo que permitió determinar el número suficiente de linfocitos y macrófagos antracóticos.

Los pacientes de la serie que requirieron mediastinoscopia diagnóstica correspondieron a un 7\%, específicamente en lesiones no tumorales malignas. Estos resultados son comparables con los publicados en la literatura mundial; por ejemplo Gilbert y colaboradores (8) publicaron un $68,1 \%$ de diagnóstico en sala e incrementaron su rendimiento al $79,7 \%$ en el bloque celular; vale anotar que la calidad de la muestra y la pericia del neumólogo intervencionista influyen en los porcentajes de diagnóstico. En cuanto al diagnóstico de lesiones neoplásicas malignas (carcinoma de células no pequeñas-CPCNP), se observó una rentabilidad diagnóstica del 91\% y una exactitud diagnóstica del $97 \%$, datos semejantes a los publicados en la literatura, que en CPCNP corresponde a una rentabilidad diagnóstica del 86,6\% y exactitud diagnóstica del $94,8 \%$ (8-10). También conviene aclarar que es importante el entrenamiento del patólogo en patología pulmonar y específicamente en citopatología pulmonar para EBUS, donde las condiciones morfológicas celulares varían al utilizar la coloración de Diff-Quick y se presentan dificultades en la determinación de células linfoides aplastadas vs. células neoplásicas de carcinomas de células pequeñas y células malignas sueltas. Otro inconveniente al utilizar la tinción de Diff-Quick es la diferenciación entre cambios reactivos y metaplasia del epitelio bronquial, células de los centros germinales, linfocitos grandes y macrófagos e inflamación granulomatosa donde existen diagnósticos diferenciales difíciles como tuberculosis, sarcoidosis y lesiones inflamatorias o malignas.

A continuación se exponen algunos de los casos estudiados en la institución, en los que se hace crucial el concepto de precisión y trabajo multidiscipli- 
nario en la práctica médica. Se explotaron ventajas como la retroalimentación inmediata de la calidad de la muestra, la evaluación de la cantidad y calidad del material, la preparación de muestras tisulares y los bloques celulares de alta calidad, así como la determinación de un triage diagnóstico individualizado que permitió aprovechar las ventajas de la medicina personalizada en los casos de cáncer pulmonar.

\section{Caso 1}

Paciente de sexo masculino, de 70 años, fumador activo, con tos crónica y lesión nodular en LII, adenopatías en ventana aorto-pulmonar e hiliares izquierdas, metabólicamente activas en la PET, a quien se le realizó EBUS-TBNA en la estación $10 \mathrm{~L}$, con extendido positivo para malignidad en sala, que arrojó carcinoma escamoso primario de pulmón (figuras 4 y 5).

\section{Caso 2}

Paciente de sexo femenino, de 66 años, con antecedente de adenocarcinoma pulmonar en 2011, quien recibió tratamiento con quimioterapia y radioterapia, en seguimiento actual y consultó por

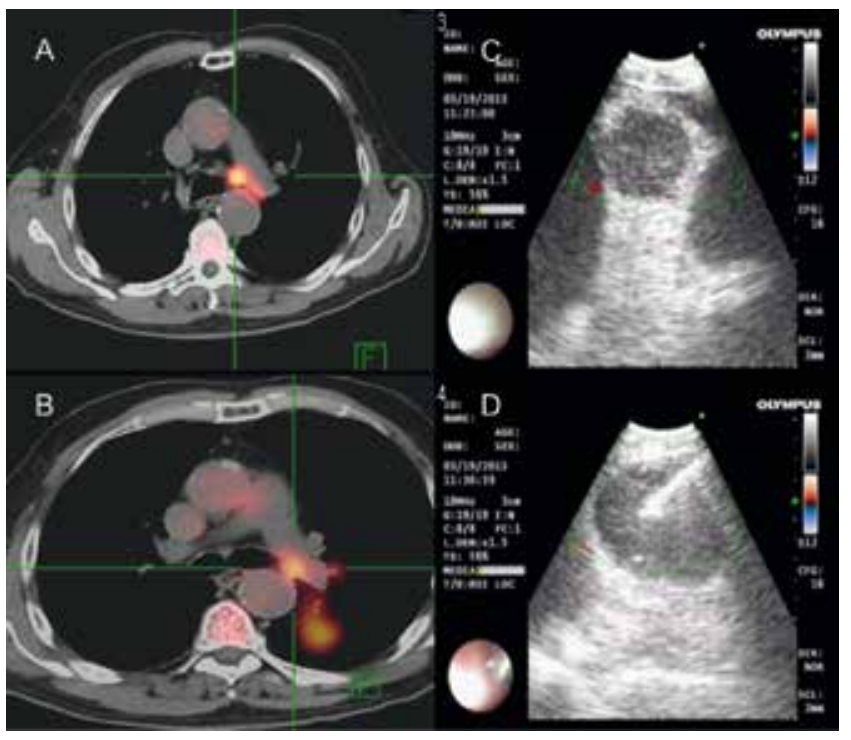

Figura 4. A, B. PET con captación metabólicamente activa en la lesión en LIl y en estaciones 10 y $11 \mathrm{~L}$. C. Ganglio identificado por medio de ultrasonido. D. Punción del ganglio en tiempo real; estación 10L.

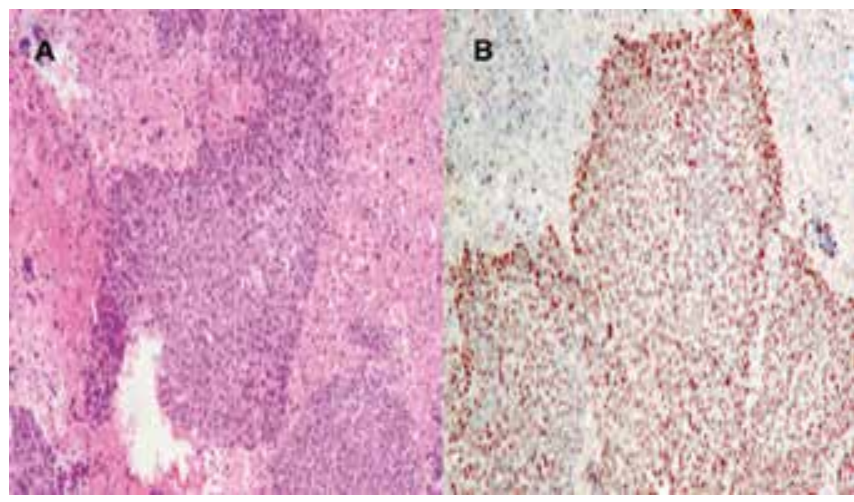

Figura 5. A. Coloración de H\&E. Bloque celular del material obtenido por EBUS. Carcinoma de células escamosas metastásico al ganglio linfático, con abundante material necrótico acompañante. B. Técnica de inmunohistoquímica. Gen p63 positivo nuclear en las células tumorales escamosas.

tos y disnea leve. Se encontró lesión paratraqueal derecha y se realizó EBUS-TBNA, que confirmó recaída tumoral con mutación del factor de crecimiento epidérmico (EGFR) positivo, más estudio de translocaciones del gen EML4-ALK negativo (figuras 6 y 7 ).

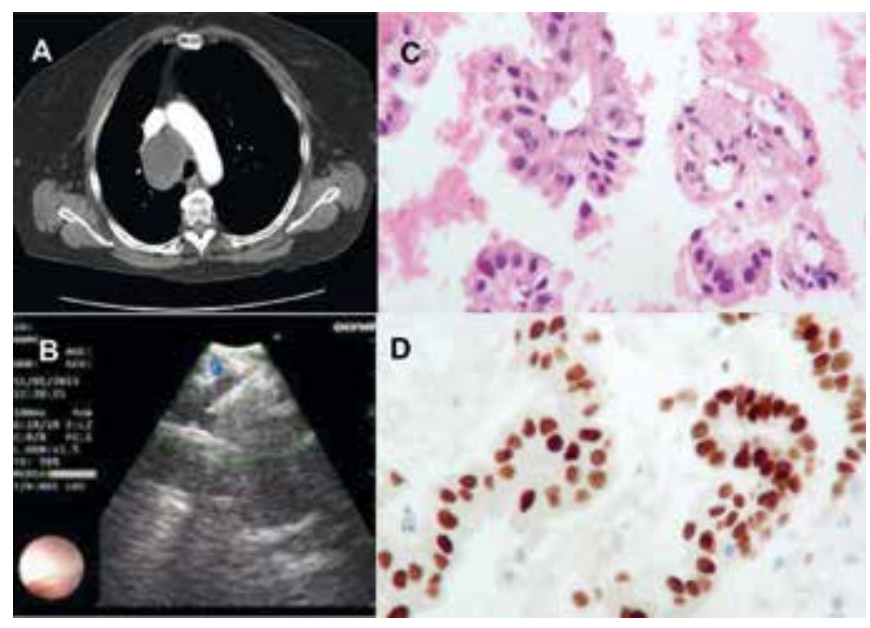

Figura 6. A. Tomografía axial computarizada en la que se aprecia lesión paratraqueal derecha. B. Punción de la lesión en tiempo real. C. Coloración de H\&E. Bloque celular. Diagnóstico de adenocarcinoma primario pulmonar. D. Técnica de inmunohistoquímica. Proteína del Gen TTF-1 positivo nuclear en las células tumorales del adenocarcinoma primario del pulmón.

Revista Colombiana de Neumología Vol 27 No 1 | 2015 


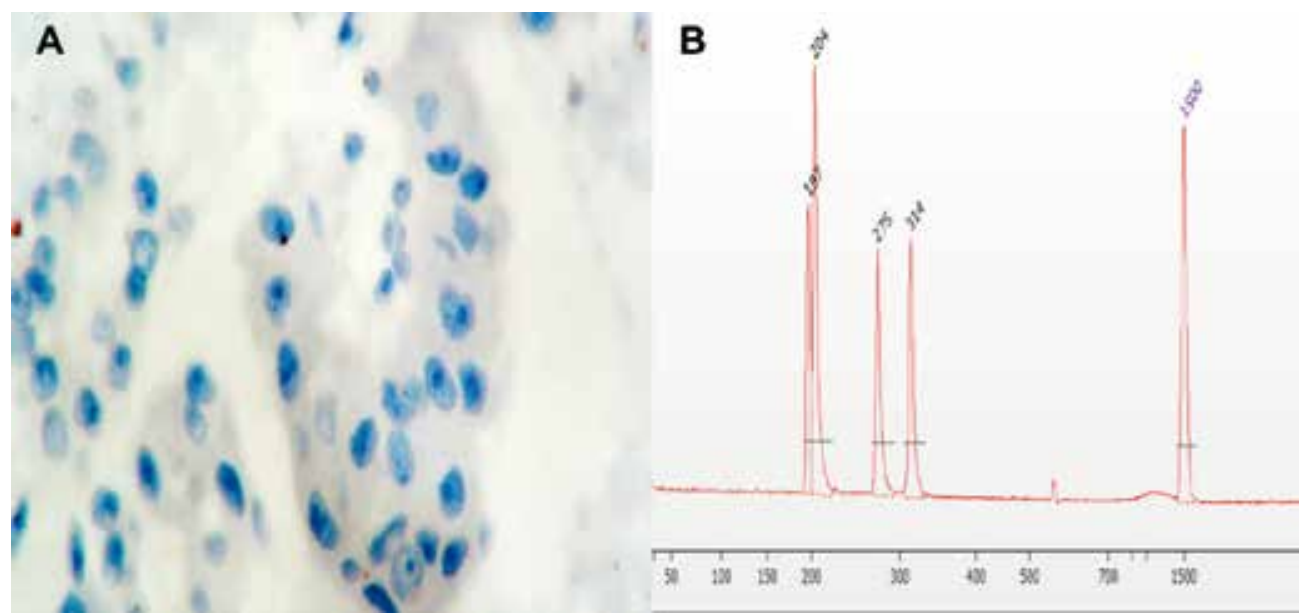

Figura 7. A. Técnica de inmunohistoquímica. Proteinacinasa para el gen EML4-ALK negativa (D5F3). B. Estudio de la mutación del gen EGFR. Extracción de ADN en el bloque obtenido durante el EBUS. Bioanalyzer 2100 (Agilent $^{\circledR}$ ) Electroforesis por microfluidos. Deleción de 15 pb en el exón 19 del gen EGFR, estado mutado (positivo).

\section{Caso 3}

Paciente de sexo femenino, de 54 años, con historia de espondilitis anquilosante con HLB27 positivo, tos crónica y adenopatía subcarinal en estudio. Se realizó EBUS-TBNA y se halló material inflamatorio con linfocitos maduros, pigmento antracótico, negativo para malignidad y PCR negativa para TBC (figura 8).

\section{Caso 4}

Paciente de sexo masculino, exfumador, quien consultó con historia de cefalea frontal y hemiparesia derecha. Se hallaron lesiones cerebrales sugestivas de metástasis. En los estudios de extensión se encontró lesión que abrazaba el bronquio fuente izquierdo con atelectasia parcial, mientras que en la fibrobroncoscopia no se observó lesión endobronquial. Se realizó EBUS-TBNA que documentó tumor de celulas pequeñas (figura 9).

\section{Caso 5}

Paciente de sexo maculino, 33 años, con historia de fiebre prolongada, pérdida de peso y lesión hiliar izquierda, más múltiples estudios negativos. Se realizó EBUS-TBNA que mostró células suges- tivas de síndrome linfoproliferativo, el cual se clasifica mediante la evaluación de la arquitectura del tejido y por tanto a través de biopsia de la lesión por toracoscopia (figura 10).

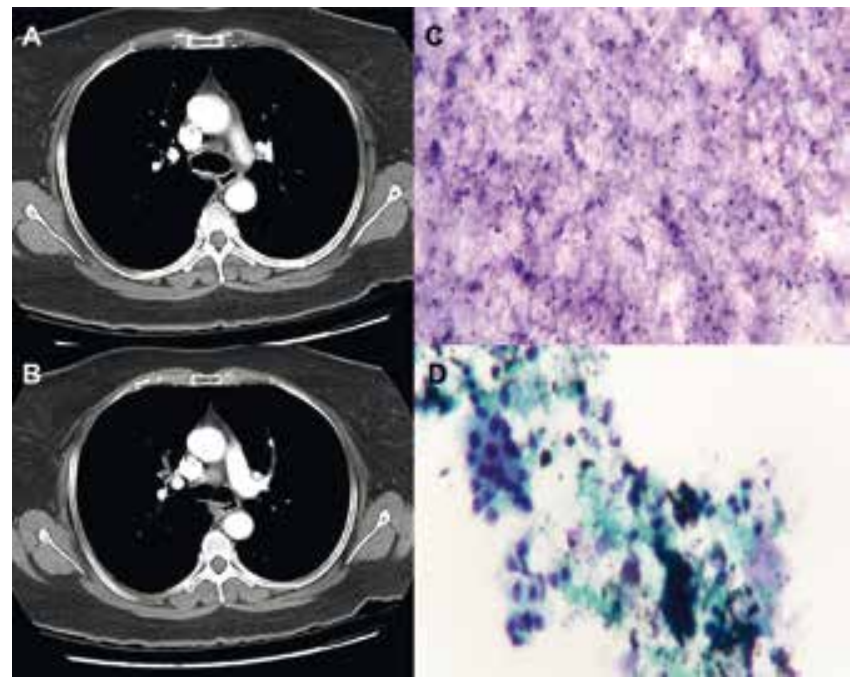

Figura 8. A, B. Tomografía axial computarizada de tórax donde se aprecian las adenopatías pre y subcarinales. C. Coloración de Diff-Quick con presencia de linfocitos maduros 50 x CAP; muestra adecuada del ganglio linfático. D. Coloración de Papanicolaou. Citología en base líquida (BD CytoRich) linfocitos maduros, histiocitos y pigmento antracótico. Negativo para malignidad. 


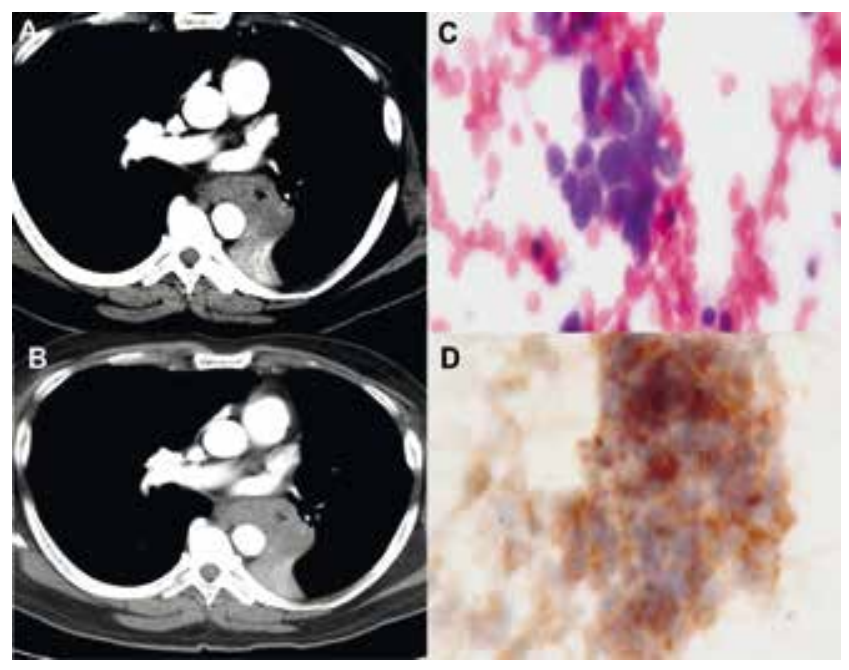

Figura 9. A, B. TAC de tórax con lesión que rodea el BFI con atelectasia parcial. C. Coloración de H\&E. Bloque celular obtenido por EBUS. Carcinoma de célula pequeña. D. Técnica de inmunohistoquímica. Positividad granular citoplasmática para cromogranina $A$ en la que se demuestra su linaje neuroendocrino.

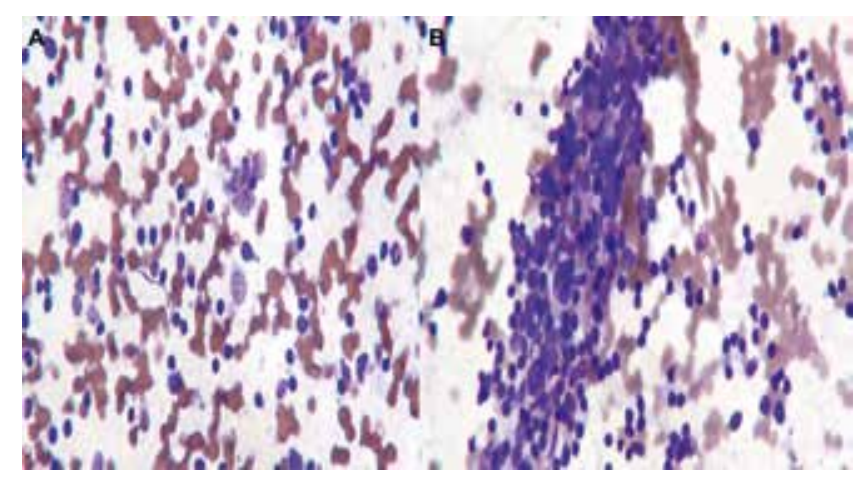

Figura 10. A. Coloración de Diff-Quick. Linfocitos maduros y linfocitos de mayor tamaño de características neoplásicas. B. Coloración de Diff-Quick. Material linfoide abundante, con el que se descarta patología neoplásica epitelial o presencia de células gigantes o necrosis. Alta sospecha de síndrome linfoproliferativo.

\section{Conclusiones}

- El EBUS-TBNA es un procedimiento eficiente en el estudio de lesiones mediastinales, paratraqueales, peribronquiales y en la estadificación y re-estadificación del cáncer pulmonar.

- En el EBUS-TBNA bien planeado, secuencial y estandarizado es posible diagnosticar y estadificar el cáncer pulmonar, en especial si se acompaña del estudio citopatológico en sala ROSE.
- El papel del patólogo intervencionista es crucial en el diagnóstico, tratamiento y pronóstico de las diferentes patologías pulmonares neoplásicas y no neoplásicas.

- La retroalimentación inmediata en la sala permite mejorar la técnica intervencionista, así como evaluar de forma precoz la calidad y cantidad de la muestra, y a su vez disponer de preparaciones y bloques celulares de alta calidad.

- Uno de los puntos más sensibles de asistir a la sala (ROSE) es poder realizar el triage en la toma de decisiones para estudios extendidos como citometría de flujo, cultivos microbiológicos y pruebas moleculares para microbiología, genética molecular y patología molecular.

\section{Bibliografía}

1. Medford AR, Bennett JA, Free CM, Agrawal S. Endobronchial ultrasound guided transbronchial needle aspiration. Postgrad Med J. 2010;86:106-15.

2. Kokkonouzis I, Strimpakos AS, Lampaditis I, Tsimpoukis S, Syrigos KN. The role of endobronchial ultrasound in lung cancer diagnosis and staging: a comprehensive review. Clin Lung Cancer. 2012;13:408-15.

3. Detterbeck FC, Jantz MA, Wallace M, Vansteenkiste J, Silvestri GA. Invasive mediastinal staging of lung cancer: ACCP evidence based clinical practice guidelines ( $2^{\text {nd. }}$ edition). Chest. 2007;132:202S-20.

4. Krasnik M, Vilmann P, Larsen SS, Jacobsen GK. Preliminary experience with a new method of endoscopic transbronchial real time ultrasound guided biopsy for diagnosis of mediastinal and hilar lesions. Thorax. 2003; 58:1083-6.

5. Aldeyturriaga J, Meléndez R, López R, Tavera E. Broncoscopia intervencionista. Arch Bronconeumol. 2010;46(Supl 6):3-7.

6. VanderLaan P, Wang H, Majid A, Folch E. Endobronchial Ultrasound-Guided Transbronchial Needle Aspiration (EBUSTBNA): An Overview and Update for the Cytipathologist. Cancer Cytopathol. 2014;122(8):561-76.

7. Louis DN, Virgin HW IV, Asa SL. "Next generation" pathology and laboratory medicine. Arch Pathol Lab Med. 2011;135(12):1531-2.

8. Gilbert S, Wilson DO, Christie NA, Pennathur A, Luketich JD, Landreneau RJ. Endobronchial ultrasound as a diagnostic tool in patients with mediastinal lymphadenopathy. Ann Thorac Surg. 2009;88(3):896-902.

9. Monaco SE, Schuchert MJ, Khalbuss WE. Diagnostic difficulties and pitfalls in rapid on-site evaluation of endobronchial ultrasound guided fine needle aspiration. Cytojournal.2010;7:9.

10. Sun W, Song K, Zervos M, Pass H, Cangiarella J, Bizekis C. The diagnostic value of endobronchial ultrasound-guided needle biopsy in lung cancer and mediastinal adenopathy. Diagn Cytopatho. 2010;38(5):33-42. 